\title{
Aberrometry: The Past, Present, and Future of Optometry
}

A berrometry of the human eye has progressed rapidly from research tool to clinical application. To appreciate how rapidly the field of clinical aberrometry is evolving, consider the following quotation from the editorial introduction to the 1997 Optometry and Vision Science feature issue on visual optics research.

"Contemporary visual optics research is changing our mindset, our way of thinking about the optical system of the eye, and in the process is re-defining the field of visual optics. In the past, optical imperfections of the eye were conceived as simple refractive errors - defocus, astigmatism, and perhaps a bit of prism. In the future, optical imperfections of the eye will be conceived in a comprehensive theoretical framework which expresses the combined effect of all the eye's optical imperfections as a two-dimensional aberrations map. When treated as a mathematical function, the aberration map may be used to compute image quality on the retina for simple points of light, for clinical test targets, or any complex object in the real world. Such computations will become routine optometric tools of the future for predicting the visual benefit to the patient of a full aberration correction. The aberration map will also become a prescription for the ideal correcting optics provided by a new kind of contact lens, spectacle lens, inter-ocular lens, or by refractive surgery."

The future has arrived! In this issue of Optometry and Vision Science, readers will learn of new discoveries that expand our basic understanding of aberrometry and the optical quality of the eye. Next month our focus shifts to clinical applications in which aberrometry is used to guide and evaluate correction of aberrations with refractive surgery and contact lenses.

What is driving this explosion of interest in aberrometry? The answer is twofold: timing and technology. The rapid growth in popularity of refractive surgery as a means of correcting refractive errors has generated a critical need for technologies for evaluating the optical outcome of surgical treatments. Initial concerns about the surgical introduction of unwanted aberrations were quickly replaced by optimistic predictions that LASIK technology could be used to correct the eye's natural aberrations, thereby achieving an optically perfect eye and super-normal vision. At the same time, designers of contact lenses and intra-ocular lenses were also dreaming of correcting the eye's aberrations. These exciting clinical developments produced a real need for patient-friendly aberrometers. Thus, the timing was perfect for commercializing a variety of technologies that had been developed in research laboratories around the world for measuring aberrations.

During the past 2 years a variety of clinical instruments has become available for routine measurement of the eye's aberrations. Although each instrument incorporates a unique design concept, almost all are based on the $17^{\text {th }}$ century principle of Scheiner's disk. $^{2}$ Scheiner's original drawing, published in 1619 in his treatise on the eye, is reproduced in Figure 1. It shows an opaque card with two pinhole apertures $B, C$ used to isolate a pair of rays entering the eye. If the point source $A$, which produced these rays, lies at the eye's far point, then the rays will intersect at a common point on

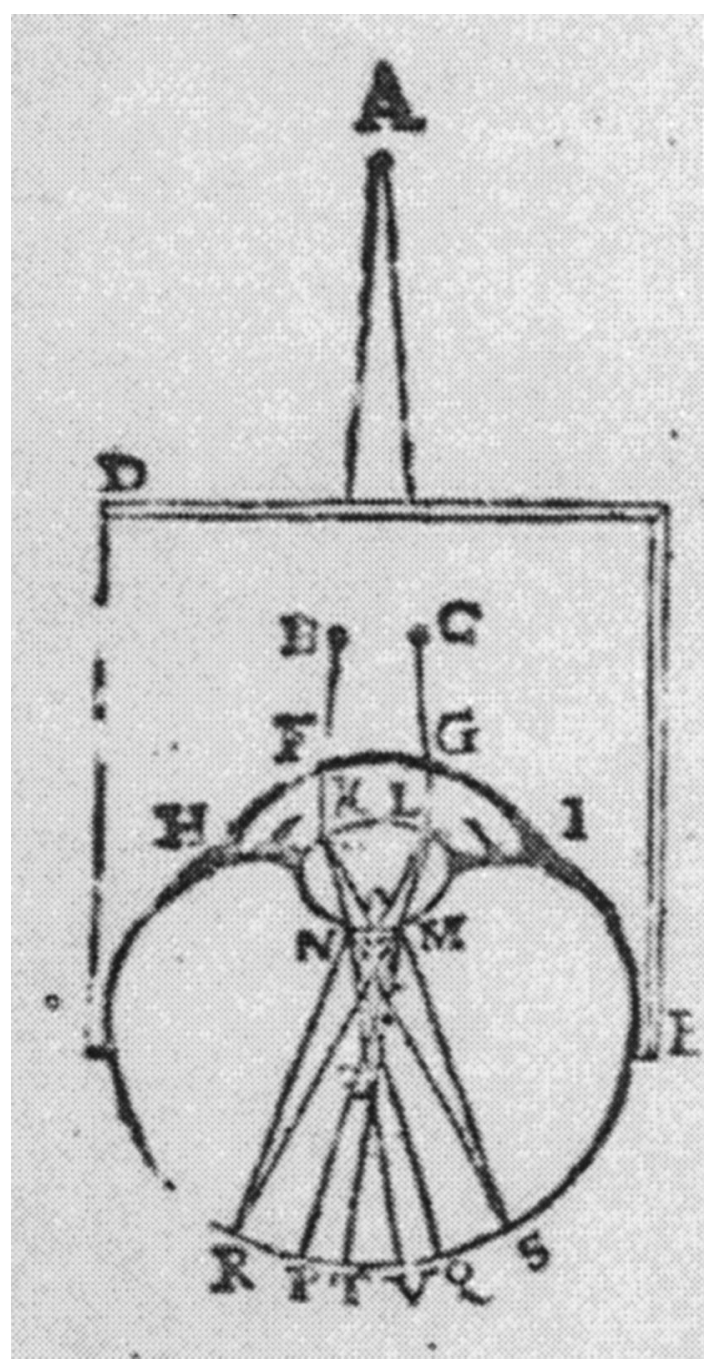

FIGURE 1.

Scheiner's original drawing, published in 1619 in his treatise on the eye. 


\section{GUEST EDITORIAL}

the retina and the patient will report seeing a single spot of light. However, if the source point is located at some axial distance other than the far point, the rays will strike different retinal locations and the observer will report seeing double. Thus Scheiner's disk can be used as a simple optometer by adjusting the point source axially until single vision is obtained, thus locating the eye's far point and simultaneously determining its spherical refractive error.

Modern aberrometers expand upon Scheiner's principle in a variety of ways. For example, the spatially-resolved refractometer creates virtual pinhole apertures that systematically move across the eye's pupil as the patient continuously adjusts the angle of incoming rays of light to compensate for their own aberrations. By recording the amount of compensation needed at each pupil location, the instrument can reconstruct the aberration map of the patient's eye. Another example is the Shack-Hartmann aberrometer, which reverses the direction of light by creating a light source on the retina with focused laser beam. Light reflected from the retina out of the eye passes through multiple apertures formed by an array of tiny lenses. This lens array subdivides the light into multiple beams that are focused into an array of spots recorded by a video camera. Mathematical analysis of the displacement of each spot from the optical axis of that lens that produced the spot yields a detailed aberration map of the eye.

Regardless of how the aberration map is generated, the interpretation is the same. Each point on the map indicates the optical distance from the eye's far point to the patient's retina through the corresponding point in the pupil, relative to the optical distance through the center of the pupil. If the optical distance is the same for all points in the pupil, then the map is flat and the eye is optically perfect. Thus, to correct all of the eye's optical imperfections requires that the aberration map be flattened. This is the goal of wavefront-guided designs of contact lenses, intra-ocular lenses, and custom refractive surgery for aberration correction. Aberrometry thus emerges as the essential clinical instrument for planning sophisticated optical treatments and measuring optical outcome. For this reason aberrometry is not only firmly rooted in optometry's past, and vigorous in the present, it is the optometer of the future. As George Woo, Dean of the Faculty of Health \& Social Sciences at The Hong Kong Polytechnic University, remarked recently, "when optometrists of the future replace optometers with aberrometers, they may very well become known as aberrometrists!" Indeed, optometrists have a well-deserved reputation for expertise in visual optics. Early adoption of aberrometry into routine clinical practice will only enhance that reputation.

\section{REFERENCES}

1. Thibos LN. The new visual optics. Optom Vis Sci 1997;74:465-6.

2. Scheiner C. Oculus. Innspruk, 1619;113.
Larry N. Thibos

Bloomington, Indiana

Raymond A. Applegate

Houston, Texas

Susana Marcos

Madrid, Spain 